\title{
Combined therapy with EGFR TKI and gambogic acid for overcoming resistance in $E G F R$-T790M mutant lung cancer
}

\author{
CHENGDE WANG $^{1,2}$, WEI WANG ${ }^{2}$, CHAOYANG WANG $^{2}$, YIJUN TANG $^{2}$ and HUI TIAN ${ }^{1}$ \\ ${ }^{1}$ Department of Thoracic Surgery, Qi Lu Hospital, Shandong University, Jinan, Shandong 250012; \\ ${ }^{2}$ Department of Thoracic Surgery, Yantai Yuhuangding Hospital, Yantai, Shandong 264000, P.R. China
}

Received September 11, 2014; Accepted June 22, 2015

DOI: $10.3892 / \mathrm{ol} .2015 .3599$

\begin{abstract}
Although patients with non-small cell lung cancer (NSCLC) experience an initial response to the epidermal growth factor receptor (EGFR) tyrosine kinase inhibitor gefitinib, those individuals with activating mutations in EGFR develop resistance. Gambogic acid (GA), a polyprenylated xanthone, has strong antitumor activities. In the present study, the therapeutic efficacy of gefitinib with GA was evaluated in a gefitinib-resistant NSCLC model. The NCI-H1975 cell line with EGFR-T790M mutation was subcutaneously injected into immunocompromised mice. The mice were randomly assigned to receive treatment with gefitinib, GA, gefitinib plus GA, or vehicle for 4 weeks, then all mice were sacrificed and their tumor tissues were subjected to caspase activity detection and western blot analysis. Gefitinib and GA alone slightly inhibited the tumor growth of NCI-H1975. However, the combined treatment significantly enhanced their antitumor effects, without any marked adverse events. In addition, gefitinib plus GA enhanced the level of apoptosis in the tumor tissues. Western blot analysis also revealed that the combination treatment reduced the phosphorylation level of AKT, MEK1/2 and ERK1/2, while an increased expression ratio of $\mathrm{Bax} / \mathrm{Bcl}-2$ was observed. In the current study, gefitinib in combination with GA resulted in antitumor growth in the EGFR-T790M secondary mutation NCI-H1975 tumor model due to an enhanced apoptotic effect. This novel therapeutic strategy may be a practical approach for the treatment of patients who show gefitinib resistance.
\end{abstract}

\section{Introduction}

Lung cancer presents with the characteristics of frequent aberrance in driver genes, particularly the epidermal growth factor receptor (EGFR) gene (1). Non-small cell lung cancer

Correspondence to: Dr Hui Tian, Department of Thoracic Surgery, Qi Lu Hospital, Shandong University, 107 West Wenhua Road, Jinan, Shandong 250012, P.R. China

E-mail: hui_tian168@126.com

Key words: gefitinib, gambogic acid, tyrosine kinase inhibitor, non-small cell lung cancer
(NSCLC) is responsible for $\leq 80 \%$ of all cases of lung cancer, and advanced disease is commonly found at the time of diagnosis. Gefitinib and erlotinib are EGFR tyrosine kinase inhibitors (EGFR-TKI) that have exhibited marked therapeutic effects against NSCLC with activating mutations in EGFR, such as exon 19 deletions and L858R point mutations (2). However, resistance will eventually develop in all patients after varying periods of time. Acquired resistance to gefitinib is most commonly conferred upon a patient by the EGFR T790M mutation, which has been detected in $50 \%$ of NSCLC cases with acquired resistance and in cell line models that have been selected for gefitinib resistance (3). Due to the limited treatment options available for individuals with advanced lung cancer, a requirement exists for the identification of novel therapeutic strategies.

Traditional Chinese medicine is used as a component of complementary and alternative medicine in the treatment of a number of diseases (4). Garcinia hanburyi, a plant belonging to the Guttiferae family, is a small tree that is found distributed throughout India, Cambodia and the southern regions of China (5). Garcinia hanburyi exudes gamboge resin, which contains gambogic acid (GA) as its main active ingredient; GA has been introduced as an effective anticancer drug $(6,7)$. The potent anticancer activity of GA is mainly dependent on the resulting activation of the impaired apoptosis pathways via downregulation of telomerase in cancer cells (8). Furthermore, GA strongly inhibits angiogenesis via vascular endothelial growth factor suppression (9). The aim of the present study was to investigate whether a combination of gefitinib and GA administration can overcome EGFR T790M-mediated resistance in patients with NSCLC.

\section{Materials and methods}

Ethics statement. All experiments were approved by the Animal User and Ethical Committees at Shandong University (Jinan, Shandong, China).

Compound. Gefitinib was obtained from Sellech Chemicals (Houston, TX, USA). Gambogic acid was purchased from Santa Cruz Biotechnology Inc. (Dallas, TX, USA).

Cell line. The NCI-H1975 EGFR T790M mutation human NSCLC cell line was obtained from the American Type 
A

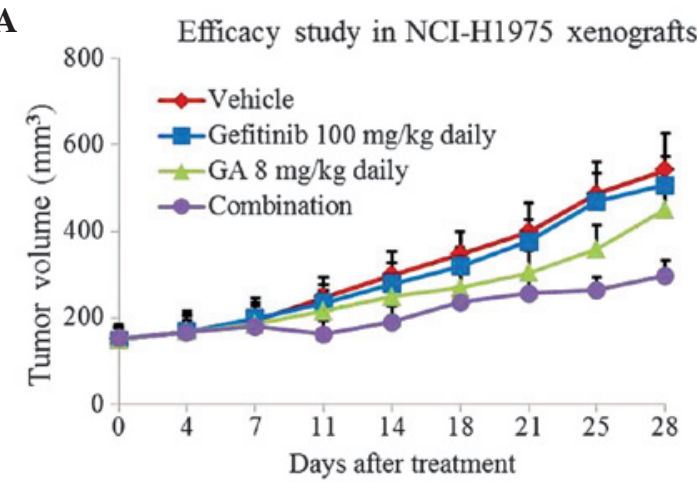

C

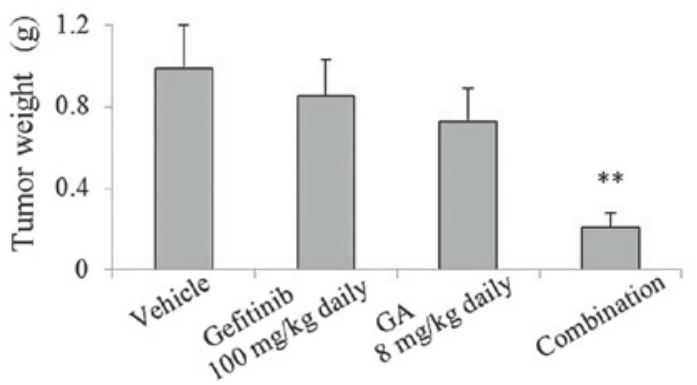

Tumor weight in NCI-H1975 xenografts

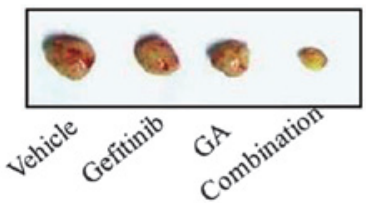

D

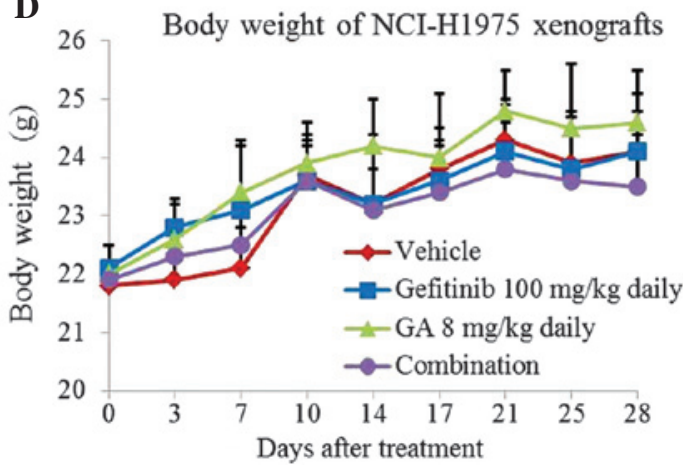

Figure 1. Antitumor activity of gefitinib or/and GA on gefitinib-resistant NCI-H1975 xenografts. Nude mice bearing NCI-H1975 tumors were orally administered $100 \mathrm{mg} / \mathrm{kg}$ gefitinib daily or/and an intravenous injection of $8 \mathrm{mg} / \mathrm{kg}$ GA daily for up to 28 days. (A) Tumor volume was measured using calipers on the indicated days. (B and C) Tumors weights were measured on day 28. (D) Body weights were measured on the indicated days. Data are presented as the mean \pm standard deviation $(n=10)$. ${ }^{* *} \mathrm{P}<0.01$ vs. control group. GA, gambogic acid.

Culture Collection (Manassas, VA, USA). The cells were cultured in RPMI 1640 medium supplemented with $10 \%$ fetal bovine serum, $100 \mathrm{U} / \mathrm{ml}$ penicillin, $100 \mathrm{mg} / \mathrm{ml}$ streptomycin and $2 \mathrm{mmol} / \mathrm{l} \mathrm{L}$-glutamine (Invitrogen Life Technologies, Carlsbad, CA, USA), and maintained at $37^{\circ} \mathrm{C}$ in a humidified atmosphere with $5 \% \mathrm{CO}_{2}$.

Efficacy study in vivo. Female, 7-8-week-old, BALB/C nude mice were purchased from Vital River Laboratories (Beijing, China). The mice were maintained in super pathogen-free conditions and housed in barrier facilities using a 12-h light/dark cycle, with food and water ad libitum. The mice were subcutaneously injected with $1 \times 10^{7}$ NCI-H1975 cells suspended in $100 \mu \mathrm{l}$ of Matrigel (BD Biosciences, Milan, Italy). The tumor volume (TV) was measured and recorded during the treatment period using the following formula: $\mathrm{TV}=$ length $\mathrm{x}$ width $^{2} / 2$. When the tumor volume reached $\sim 150 \mathrm{~mm}^{3}$, the mice were randomly divided into four groups ( $n=10$ for each group) and received normal saline (vehicle group, daily intravenous injection), gefitinib (100 mg/kg, daily oral administration), GA solution $(8 \mathrm{mg} / \mathrm{kg}$, daily intravenous injection) or a combination treatment of gefitinib and GA for 28 days. Gefitinib was dissolved in $0.1 \%$ Tween 80 prior to use.

Western blot analysis. The tumors in each group were collected $4 \mathrm{~h}$ after the last treatment with gefitinib, GA or the combination treatment on day 28 of the efficacy study. Western blotting was performed as described previously (10). Proteins $(50 \mu \mathrm{g})$ were separated by $12 \%$ SDS-PAGE and then transferred onto $0.45-\mu \mathrm{m}$ polyvinylidine fluoride membranes (Bio-Rad Laboratories, Inc., Hercules, CA, USA). The membranes were blocked in phosphate-buffered saline containing 5\% non-fat dry milk for $1 \mathrm{~h}$, then incubated at $4^{\circ} \mathrm{C}$ overnight with the following rabbit anti-human monoclonal primary antibodies: AKT (dilution, 1:1,000; catalog no. 4685), phosphorylated (p)-AKT (Ser473; dilution, 1:1,000; catalog no. 4058), ERK1/2 (dilution, 1:1,000; catalog no. 4695), p-ERK1/2 (Thr202/Tyr204; dilution, 1:1,000; catalog no. 14474), MEK1/2 (dilution, 1:1,000; catalog no. 13033), p-MEK1/2 (dilution, 1:1,000; catalog no. 2338), Bax (dilution, 1:1,000; catalog no. 5023) and Bcl-2 (dilution, 1:1,000; catalog no. 2870) (Cell Signaling Technology, Inc., Danvers, MA, USA). This was followed by incubation with horseradish peroxidase-conjugated anti-rabbit secondary antibody (EMD Millipore, Billerica, MA, USA).

Caspase activity assay. Caspase-3, 8 and 9 activity was evaluated by fluorometric detection of apoptosis with caspase-3 (cat. no. KA0740),-8 (cat. no. KA0756) and -9 (cat. no. KA0761) colorimetric protease kits (Abnova, Walnut, CA, USA) according to the manufacturer's instructions. Briefly, the tumor tissues were obtained and lysed in $100 \mu 1$ cell lysis buffer (Cell Signaling Technology, Inc.), and $200 \mu \mathrm{g}$ protein was incubated with $5 \mu \mathrm{l} 4 \mathrm{mM}$ pNA-conjugated substrate (DEVD- $p$ NA for caspase-3, LETD- $p$ NA for caspase- 8 and LEHD- $p$ NA for caspase-9; Abnova) at $37^{\circ} \mathrm{C}$ for $2 \mathrm{~h}$. The amount of pNA released was measured at $405 \mathrm{~nm}$ using a UV-Vis Flour spectrophotometer (CRAIC Technologies, Inc., San Dimas, CA, USA). Next, tissue lysates were 


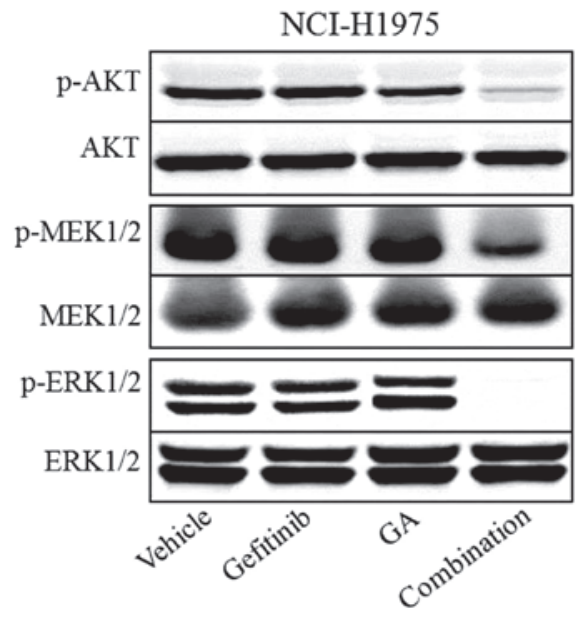

Figure 2. Effects of gefitinib or/and GA therapy on PI3K and ERK pathways. The gefitinib-resistant NCI-H1975 xenografts were treated with gefitinib or/and GA on day 28 of the efficacy study. Tumor tissues were then collected $4 \mathrm{~h}$ later to detect levels of phosphorylated (p)-AKT (S473)/AKT, p-ERK (T202/Y204)/ERK (S240/244) and p-MEK/MEK. GA, gambogic acid.

$\mathbf{A}$

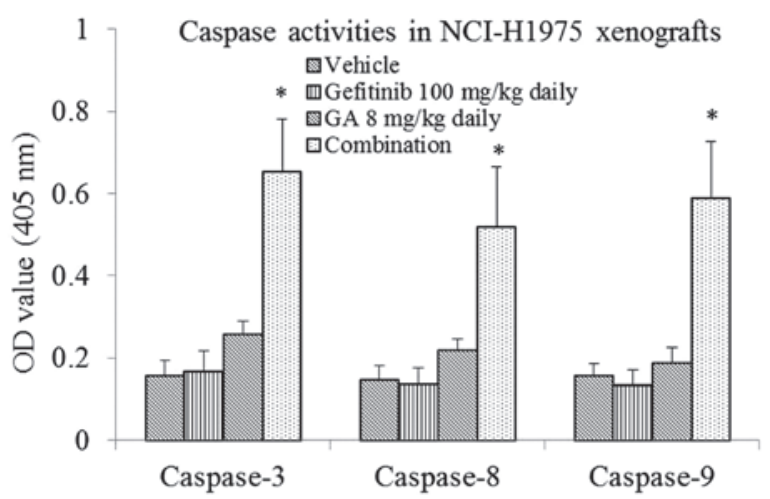

B

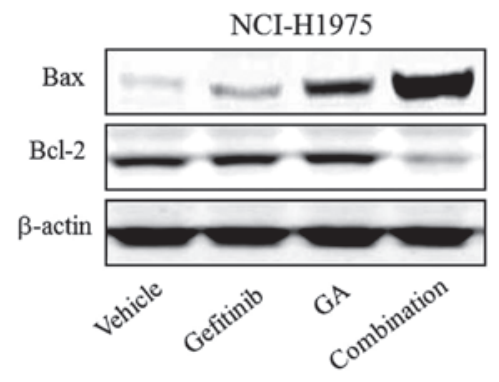

Figure 3. Gefitinib in combination with GA induces apoptosis in gefitinib-resistant NCI-H1975 xenografs. NCI-H1975 xenografts were treated with gefitinib or/and GA on day 28 of the efficacy study. (A) Tumor tissues in each group $(n=10)$ were measured by caspase colorimetric protease kits. ${ }^{*} \mathrm{P}<0.05$ vs. vehicle. (B) The expression of Bax and Bcl-2 was analyzed by western blotting (B). GA, gambogic acid; OD, optical density.

centrifuged at 3,000 x g, resuspended in $100 \mu \mathrm{l}$ apopain lysis buffer and vortexed gently. The suspensions were frozen and thawed 4-5 times using liquid nitrogen and a $37^{\circ} \mathrm{C}$ water bath, respectively.

Statistical analysis. Data are expressed as the mean \pm standard deviation. InStat, version 1.14 (GraphPad Software, Inc. San Diego, CA, USA) was used for statistical analysis, and a two-way unweighted mean analysis of variance test was used to determine the statistical significance. $\mathrm{P}<0.05$ was considered to indicate a statistically significant difference.

\section{Results}

Gefitinib in combination with GA treatment has a synergistic effect on gefitinib-resistant NCI-H1975 tumor growth in vivo. In order to investigate the inhibitory effect of gefitinib or/and GA on tumor growth in vivo, gefitinib, GA and gefitinib plus GA were used to treat NCI-H1975 xenografts for 4 weeks. As presented in Fig. 1A-C, treatment with gefitinib or GA for 4 weeks slightly inhibited tumor growth in the NCI-H1975 xenografts. However, more marked inhibition was caused by the combined treatment, which resulted in a $\sim 70 \%$ reduction in tumor growth $(\mathrm{P}=0.008$; Fig. $1 \mathrm{C})$. The mice tolerate the single-agent and combination treatments well, with no weight loss or other signs of acute or delayed toxicity (Fig. 1D).

Effect of the combined treatment on PI3K and ERK signaling pathways in gefitinib-resistant NCI-H1975 xenografts. Western blot analysis was used to assess the effect of the two compounds on downstream molecules of the PI3K and ERK pathways. The results showed that p-AKT, p-MEK1/2 and p-ERK1/2 appeared to be inhibited by gefitinib and GA combination treatment, whereas the total protein levels of AKT, MEK1/2 and ERK1/2 remained unchanged in each of the groups (Fig. 2). Western blot analysis also showed that single-agent treatment with gefitinib or GA only exhibited a slightly inhibitory effect on the phosphorylation of AKT, MEK1/2 and ERK1/2 in the NCI-H1975 xenografts.

Effect of combined treatment-induced apoptosis in gefitinib-resistant NCI-H1975 xenografts. To study whether gefitinib in combination with GA would induce apoptosis in the gefitinib-resistant NCI-H1975 xenografts, the activity of caspase-3, 8 and 9 were measured by colorimetric assay. As presented in Fig. 3A, the results indicated that the gefitinib and GA single-agent treatments exhibited no significant effect on caspase-3, 8 and 9 activity in the NCI-H1975 xenografts ( $\mathrm{P}>0.05$ compared with the vehicle), whereas the combined treatment led to a significant increase $(\mathrm{P}<0.05$ compared with the vehicle).

The results of western blotting also showed that the expression of Bax was upregulated, whereas the expression of Bcl-2 was downregulated by the combination treatment (Fig. 3B).

\section{Discussion}

The resistance to the EGFR-TKIs gefitinib and erlotinib ultimately develops in all patients with metastatic EGFR mutant lung cancer. The most commonly observed mechanism for this involves the acquisition of cells harboring a second-site mutation, T790M (11). Irreversible EGFR-TKIs, such as BIBW2992, that can overcome the T790M-mediated resistance to gefitinib have been developed (12), however, clinical trials have failed to show that monotherapy with such irreversible EGFR-TKIs leads to any benefit in those patients with NSCLC refractory to gefitinib (13). Thus, effective therapies for these patients are urgently required. Certain studies have demonstrated the significant anti-proliferative 
and pro-apoptotic effects of GA on a range of human cancer cell types in vitro and in vivo $(14,15)$. In the current study, gefitinib in combination with GA was found to have a synergistic inhibitory effect on gefitinib-resistant NCI-H1975 tumor growth, whereas single-agent treatment with gefitinib or GA only exhibited a slight inhibitory effect on tumor growth.

It is established that apoptosis caused by mitochondria is involved in the activation of caspases and Fas-associated death domain protein activation. In the former case, caspase-9 is activated by mitochondrial permeability transitions $(\psi \mathrm{m})$, which are mediated by cytochrome $c$ release and a reduction in the Bcl-2/Bax ratio (16). In the latter case, Fas-associated death domain protein activates caspase- 8 , which in turn activates downstream executioners caspase-3 or -7 . Xu et al (17) reported that GA causes the induction of mitochondria-dependent apoptosis via Bcl-2 and Bax modulation in mantle cell lymphoma JeKo-1 cells. However, in the current study, single-agent treatment with GA could not induce apoptosis in the NCI-H1975 xenografts. It was notable that the combined treatment caused significantly increased levels of caspase 3,8 and 9 activity, and that an increased expression ratio of Bax/ Bcl-2 was also observed in the tumor tissues. The detailed mechanisms behind this require further investigation.

In conclusion, the enhancement of apoptosis caused by treatment with the combination of gefitinib and GA was effective in the suppression of gefitinib-resistant tumor growth caused by a EGFR T790M secondary mutation. These findings provide a promising future strategy for the treatment of gefitinib-resistant NSCLC.

\section{References}

1. She J, Yang P, Hong Q and Bai C: Lung cancer in China: Challenges and interventions. Chest 143: 1117-1126, 2013.

2. Weir HK, Thun MJ, Hankey BF, Ries LA, Howe HL, Wingo PA, Jemal A, Ward E, Anderson RN and Edwards BK: Annual report to the nation on the status of cancer, 1975-2000, featuring the uses of surveillance data for cancer prevention and control. J Natl Cancer Inst 95: 1276-1299, 2003.

3. Shimamura T, Li D, Ji H, Haringsma HJ, Liniker E, Borgman CL, Lowell AM, Minami Y, McNamara K, Perera SA, et al: Hsp90 inhibition suppresses mutant EGFR-T790M signaling and overcomes kinase inhibitor resistance. Cancer Res 68: 5827-5838, 2008.

4. Yang H, Chen D, Cui QC, Yuan X and Dou QP: Celastrol, a triterpene extracted from the Chinese 'Thunder of God Vine' is a potent proteasome inhibitor and suppresses human prostate cancer growth in nude mice. Cancer Res 66: 4758-4765, 2006.
5. Sukpondma Y, Rukachaisirikul V and Phongpaichit S: Antibacterial caged-tetraprenylated xanthones from the fruits of Garcinia hanburyi. Chem Pharm Bull (Tokyo) 53: 850-852, 2005.

6. Wu ZQ, Guo QL, You QD, Zhao L and Gu HY: Gambogic acid inhibits proliferation of human lung carcinoma SPC-A1 cells in vivo and in vitro and represses telomerase activity and telomerase reverse transcriptase mRNA expression in the cells. Biol Pharm Bull 27: 1769-1774, 2004.

7. Guo QL, Lin SS, You QD, Gu HY, Yu J, Zhao L, Qi Q, Liang F, Tan $\mathrm{Z}$ and Wang $\mathrm{X}$ : Inhibition of human telomerase reverse transcriptase gene expression by gambogic acid in human hepatoma SMMC-7721 cells. Life Sci 78: 1238-1245, 2006.

8. Zhao L, Guo QL, You QD, Wu ZQ and Gu HY: Gambogic acid induces apoptosis and regulates expressions of Bax and $\mathrm{Bcl}-2$ protein in human gastric carcinoma MGC-803 cells. Bio Pharm Bull 27: 998-1003, 2004.

9. Yi T, Yi Z, Cho SG, Luo J, Pandey MK, Aggarwal BB and Liu M: Gambogic acid inhibits angiogenesis and prostate tumor growth by suppressing vascular endothelial growth factor receptor 2 signaling. Cancer Res 68: 1843-1850, 2008.

10. Sano T, Takeuchi S, Nakagawa T, Ishikawa D, Nanjo S, Yamada T, Nakamura T, Matsumoto K and Yano S: The novel phosphoinositide 3-kinase-mammalian target of rapamycin inhibitor, BEZ235, circumvents erlotinib resistance of epidermal growth factor receptor mutant lung cancer cells triggered by hepatocyte growth factor. Int J Cancer 133: 505-513, 2013.

11. Pao W, Miller VA, Politi KA, Riely GJ, Somwar R, Zakowski MF, Kris MG and Varmus H: Acquired resistance of lung adenocarcinomas to gefitinib or erlotinib is associated with a second mutation in the EGFR kinase domain. PLoS Med 2: e73, 2005.

12. Li D, Ambrogio L, Shimamura T, Kubo S, Takahashi M, Chirieac LR, Padera RF, Shapiro GI, Baum A, Himmelsbach F, et al: BIBW2992, an irreversible EGFR/HER2 inhibitor highly effective in preclinical lung cancer models. Oncogene 27: 4702-4711, 2008.

13. Wong KK, Fracasso PM, Bukowski RM, Lynch TJ, Munster PN, Shapiro GI, Jänne PA, Eder JP, Naughton MJ, Ellis MJ, et al: A phase I study with neratinib (HKI-272), an irreversible pan ErbB receptor tyrosine kinase inhibitor, in patients with solid tumors. Clin Cancer Res 15: 2552-2558, 2009.

14. Zhao Q, Yang Y, Yu J, You QD, Zeng S, Gu HY, Lu N, Qi Q, Liu W, Wang XT, et al: Posttranscriptional regulation of the telomerase hTERT by gambogic acid in human gastric carcinoma 823 cells. Cancer Lett 262: 223-231, 2008.

15. Pandey MK, Sung B, Ahn KS, Kunnumakkara AB, Chaturvedi MM and Aggarwal BB: Gambogic acid, a novel ligand for transferrin receptor, potentiates TNF-induced apoptosis through modulation of the nuclear factor-kappaB signaling pathway. Blood 110: 3517-3525, 2007.

16. Adrain C, Creagh EM and Martin SJ: Defying death: Showing Bcl-2 the way home. Nat Cell Biol 5: 9-11, 2003.

17. Xu J, Zhou M, Ouyang J, Wang J, Zhang Q, Xu Y, Xu Y, Zhang Q, Xu X and Zeng H: Gambogic acid induces mitochondria-dependent apoptosis by modulation of Bcl-2 and Bax in mantle cell lymphoma JeKo-1 cells. Chin J Cancer Res 25: 183-191, 2013. 